\title{
Characteristics and contraceptive outcomes of women seeking medical or surgical abortion in reproductive health clinics in Cambodia
}

\author{
Chris Smith ${ }^{1,2^{*}}$, Rachel H. Scott ${ }^{2}$, Caroline Free ${ }^{2}$ and Tansy Edwards ${ }^{3}$
}

\begin{abstract}
Background: Since the approval of medical abortion in Cambodia in 2010, the proportion of women reporting an abortion has increased. We describe the characteristics and contraceptive outcomes of women seeking medical abortion compared to surgical abortion at four reproductive health clinics in Cambodia.

Methods: Secondary data analysis using data collected in the MObile Technology for Improved Family Planning (MOTIF) trial, a single blind, randomized trial of a personalized, mobile phone-based intervention designed to support post-abortion family planning in Cambodia. Baseline interviews were conducted after women had received post-abortion family planning counselling at the clinic, and follow-up interviews were conducted at 4 and 12 months. We used multivariable logistic regression to assess associations between abortion type and baseline characteristics, contraceptive uptake, repeat pregnancy and repeat abortion. We conducted an exploratory analysis to compare the timing of initiation of long-acting (LA) contraception between women having medical versus surgical abortion over the four-month post-abortion period.

Results: Of the 500 women who participated in the trial, $41 \%$ had a medical abortion. In multivariate analyses, women undergoing medical abortion were more likely to be urban residents, have a higher level of education, be undecided or not intending to use family planning, and be undecided or intending to have another child. There was no association between type of abortion and contraceptive uptake, repeat pregnancy or repeat abortion. Women who had medical abortion initiated LA contraception post abortion later than women who had surgical abortion.

Conclusions: Our results suggest women having a medical abortion in Cambodia have different baseline characteristics and had delayed uptake of contraception compared to women having a surgical abortion. However, we cannot draw conclusions on the direction of associations and causality. Further research is recommended to explore reasons for the observed findings with view to increasing access to abortion and post-abortion contraception.
\end{abstract}

Keywords: Contraception, Post-abortion family planning, Post-abortion contraception, Medical abortion, Surgical abortion

\footnotetext{
* Correspondence: christopher.smith@lshtm.ac.uk

'Graduate School of Tropical Medicine and Global Health, Nagasaki

University, Nagasaki, Japan

${ }^{2}$ Department of Population Health, London School of Hygiene \& Tropical

Medicine, London, UK

Full list of author information is available at the end of the article
}

(c) The Author(s). 2019 Open Access This article is distributed under the terms of the Creative Commons Attribution 4.0 International License (http://creativecommons.org/licenses/by/4.0/), which permits unrestricted use, distribution, and reproduction in any medium, provided you give appropriate credit to the original author(s) and the source, provide a link to the Creative Commons license, and indicate if changes were made. The Creative Commons Public Domain Dedication waiver (http://creativecommons.org/publicdomain/zero/1.0/) applies to the data made available in this article, unless otherwise stated. 


\section{Introduction}

It is estimated that globally around $25 \%$ of pregnancies resulted in induced surgical or medical abortion during the period 2010-2014, equating to an annual abortion rate of 35 per 1000 women aged 15-44. [1] In Cambodia in response to high maternal mortality, estimated at 900/ 100,000 live births, abortion laws were reformed in 1997 to allow abortion on request until the 12th week of pregnancy, and in certain circumstances during the second trimester. [2]. According to the 1997 law, abortions can only be provided by medical doctors, medical practitioners or midwives authorized by the Ministry of Health and only carried out in a hospital, health center, health clinic or maternity ward. [3] The medical abortion pack 'Medabon' containing mifepristone and misoprostol was approved by the Cambodia Ministry of Health in 2010 and made available in a restricted number of health facilities and pharmacies. [2] Medical abortion is available in clinic settings and from pharmacies and medical abortion packs have been distributed in Cambodia via social marketing and franchising programmes by non-governmental organisations such as Population Services Khmer. [4] Cambodian women also use other pharmaceutical products to induce an abortion, purchased from pharmacists, markets and drug sellers; typically unregistered combinations of mifepristone and misoprostol, sometimes described as "Chinese pills" $[4,5]$.

Sequential demographic and health surveys in Cambodia indicate that the proportion of women reporting having had an induced abortion in the previous 5 years increased from $4 \%$ in 2005 to $7 \%$ in 2014 . [3, 6, 7] Following the approval of medical abortion in 2010, the proportion of women having medical rather than surgical abortion increased: from $31 \%$ in 2010 to $47 \%$ in 2014. [3, 7]

Women seeking abortion services often have an unmet need for family planning. Post-abortion family planning (PAFP) can reduce subsequent unplanned pregnancies and repeat abortion and was identified as a high-impact practice in family planning by a USAID technical advisory group in 2012. [8] Women are at risk of pregnancy soon after abortion; studies that have examined return to ovulation post-abortion show that this can occur around 2-3 weeks following the abortion, but earlier among some women. $[9,10]$ All contraceptive methods are safe to use in the immediate post-abortion period to reduce the risk of pregnancy, with the exception of the intra-uterine device (IUD), which can be inserted at the time of surgical abortion but in the case of medical abortion should be delayed until after the abortion is complete. [1113] The type of abortion obtained may therefore affect uptake of post-abortion contraception or the contraceptive method chosen.
We describe characteristics and contraceptive outcomes of women seeking medical abortion vs. surgical abortion, using data collected in the MObile Technology for Improved Family Planning (MOTIF) trial. [14] The specific objectives are to compare: (1) baseline characteristics amongst women seeking medical versus surgical abortion in the MOTIF trial; (2) outcomes: contraception use, repeat pregnancy and abortion amongst women seeking medical versus surgical abortion in the trial; and (3) the timing of initiation of long-acting contraception between women having medical versus surgical abortion over the four-month post-abortion period.

\section{Methods}

We analysed data collected during the MOTIF trial. The trial protocol, results and process evaluation are reported in separate manuscripts. $[15,16]$ In brief, the MOTIF trial was a single blind, randomized trial of a personalized, mobile phone-based intervention designed to support post-abortion contraception. Women were recruited by research assistants from four clinics in Cambodia during May to September 2013. The clinics in Phnom Penh (2), Battambang and Siem Reap served clients from urban and rural areas. On recruitment, women were asked whether they considered their residence to be urban or rural. Women were eligible for inclusion if they were aged 18 or older, attending for an induced abortion at the MSI clinic (either surgical or medical, using licensed medication), had a mobile phone primarily for their own use, reported not wanting to become pregnant and were willing to receive automated messages about contraception. Baseline interviews were conducted after women had received post-abortion family planning counselling at the clinic for the abortion visit, and follow-up interviews were conducted at 4 and 12 months. Follow-up was $86 \%$ at 4 months and $66 \%$ at 12 months. There was no evidence of differential loss to follow-up between women receiving medical compared to surgical abortion at four or 12 months. [17]

\section{Analysis}

First, factors associated with medical versus surgical abortion in univariable analyses were identified, based on a likelihood ratio test (LRT) $p$-value $<0.1$. A multivariable model was obtained from a stepwise model building process, whereby covariates were added to the multivariable model in turn, and the goodness of fit of the model with the additional parameter was compared to the previous model using a LRT. Covariates were retained in the multivariable model if the LRT $p$-value $<$ 0.1. Covariates were removed if not associated with the outcome after adjustment for other covariates during this process. 
Table 1 Characteristics of the study sample

\begin{tabular}{|c|c|}
\hline & $\mathrm{N}$ \\
\hline \multicolumn{2}{|l|}{ Type of abortion } \\
\hline Medical & 207 \\
\hline Surgical & 293 \\
\hline \multicolumn{2}{|l|}{ Age group } \\
\hline Age $>25$ & 343 \\
\hline Age $<25$ & 157 \\
\hline \multicolumn{2}{|l|}{ Residence } \\
\hline Rural & 321 \\
\hline Urban & 179 \\
\hline \multicolumn{2}{|l|}{ Socio-economic status } \\
\hline No access to motorised transport & 65 \\
\hline Access to motorised transport & 435 \\
\hline \multicolumn{2}{|l|}{ Level of Education } \\
\hline None or primary & 196 \\
\hline Some secondary or above & 304 \\
\hline \multicolumn{2}{|l|}{ Marital status } \\
\hline Never married or living together & 29 \\
\hline Married/living together & 464 \\
\hline Divorced/separated & 7 \\
\hline \multicolumn{2}{|l|}{ \# living children } \\
\hline 1 or more & 353 \\
\hline 0 & 147 \\
\hline \multicolumn{2}{|l|}{ \# previous abortions } \\
\hline 0 & 299 \\
\hline 1 or more & 201 \\
\hline \multicolumn{2}{|l|}{ Previous contraception use } \\
\hline Yes & 299 \\
\hline No & 201 \\
\hline \multicolumn{2}{|l|}{ Occupation } \\
\hline Housewife & 102 \\
\hline Factory worker & 76 \\
\hline Entertainment worker & 27 \\
\hline Farmer & 32 \\
\hline Employed & 99 \\
\hline Self-employed/own business & 134 \\
\hline Casual work/casual labour & 3 \\
\hline Student & 17 \\
\hline Unemployed & 9 \\
\hline vOther & 1 \\
\hline \multicolumn{2}{|l|}{ Contraception decision-making ${ }^{\text {a }}$} \\
\hline Mainly woman & 96 \\
\hline Mainly husband/partner & 78 \\
\hline Joint decision & 290 \\
\hline
\end{tabular}

Phone sharing
Table 1 Characteristics of the study sample (Continued)

\begin{tabular}{|c|c|c|}
\hline & $\mathrm{N}$ & $\%$ \\
\hline Shares & 241 & 48 \\
\hline Never shares & 259 & 52 \\
\hline \multicolumn{3}{|c|}{ Disclosure of abortion to others ${ }^{b}$} \\
\hline Yes & 204 & 85 \\
\hline No & 37 & 15 \\
\hline \multicolumn{3}{|l|}{ PAFP intentions } \\
\hline Yes & 187 & 37 \\
\hline No & 42 & 8 \\
\hline Undecided & 271 & 54 \\
\hline \multicolumn{3}{|l|}{ Fertility plans } \\
\hline No more/none & 139 & 28 \\
\hline Have a/another child & 308 & 62 \\
\hline Undecided & 53 & 11 \\
\hline \multicolumn{3}{|l|}{ Phone credit } \\
\hline Sometimes & 193 & 39 \\
\hline Usually & 128 & 26 \\
\hline Always & 179 & 36 \\
\hline \multicolumn{3}{|l|}{ Randomisation } \\
\hline Intervention & 249 & 50 \\
\hline Control & 251 & 50 \\
\hline
\end{tabular}

Secondly, we assessed crude and adjusted associations between abortion method and effective contraceptive use, repeat pregnancy and abortion at 4 and 12-months using logistic regression. We define effective modern methods to be those associated with < $10 \%$ 12-month pregnancy rates, as commonly used: Oral Contraceptive (OC), injectable, implant, IUD, or permanent methods [18]. A participant was considered to be using an effective method if she reported that she: (i) was currently using the implant or IUD; (ii) had received the injectable within the previous 3 months; (iii) was sterilized (or partner was sterilized); or (iv) had taken an $\mathrm{OC}$ within $24 \mathrm{~h}$ of the interview or according to instructions. Long acting methods were defined as the IUD, implant or a permanent method. The pre-specified approach was to adjust for age, socio-economic status and residence a priori, as well whether the participant was allocated to receive the intervention or not. Access to motorised transport was used as a measure of socio-economic status, similar to the assessment of household wealth in the Cambodia DHS survey. [3] Age cut offs above and below 25 were selected to be consistent with MSI definitions of young women and our previous publications. [19] All participants who were allocated to the intervention and could be contacted received the intervention messages. Those who could not be 
Table 2 Associations with type of abortion

\begin{tabular}{|c|c|c|c|c|c|c|}
\hline & Surgical & Medical & Crude OR & LRT $p$ value & Adjusted $\mathrm{OR}^{\mathrm{a}}$ & LRT $p$ value \\
\hline & $N=293$ & $N=207$ & & & & \\
\hline Age group & & & & & & \\
\hline Age $>25$ & $213(62 \%)$ & $130(38 \%)$ & 1.00 & & & \\
\hline Age $<25$ & $80(51 \%)$ & 77 (49\%) & $1.58(1.08-2.31)$ & 0.019 & & \\
\hline Residence & & & & & & \\
\hline Rural & $216(67 \%)$ & $105(33 \%)$ & 1.00 & & & \\
\hline Urban & 77 (43\%) & $102(57 \%)$ & $2.73(1.87-3.97)$ & $<0.001$ & $2.18(1.46-3.25)$ & $<0.001$ \\
\hline Socio-economic status & & & & & & \\
\hline No access to motorised transport & $45(69 \%)$ & $20(31 \%)$ & 1.00 & & & \\
\hline Access to motorised transport & $248(57 \%)$ & $187(43 \%)$ & $1.70(0.97-2.97)$ & 0.058 & & \\
\hline Level of Education & & & & & & \\
\hline None or primary & $130(66 \%)$ & $66(34 \%)$ & 1.00 & & & \\
\hline Some secondary or above & $163(54 \%)$ & $141(46 \%)$ & $1.70(1.17-2.47)$ & 0.005 & $1.45(0.97-2.17)$ & 0.068 \\
\hline Marital status & & & & & & \\
\hline Never married or living together & $18(62 \%)$ & $11(38 \%)$ & 1.00 & & & \\
\hline Married/living together & $271(58 \%)$ & $193(42 \%)$ & $1.17(0.54-2.52)$ & 0.924 & & \\
\hline Divorced/separated & $4(57 \%)$ & $3(43 \%)$ & $1.23(0.23-6.55)$ & & & \\
\hline \# living children & & & & & & \\
\hline 1 or more & $223(63 \%)$ & $130(37 \%)$ & 1.00 & & & \\
\hline 0 & $70(48 \%)$ & 77 (52\%) & $1.89(1.28-2.78)$ & 0.001 & & \\
\hline \# previous abortions & & & & & & \\
\hline 0 & $171(57 \%)$ & $128(43 \%)$ & 1.00 & & & \\
\hline 1 or more & $122(61 \%)$ & 79 (39\%) & $0.87(0.60-1.25)$ & 0.435 & & \\
\hline Previous contraception use & & & & & & \\
\hline Yes & $180(60 \%)$ & $119(40 \%)$ & 1.00 & & & \\
\hline No & $113(56 \%)$ & $88(44 \%)$ & $1.18(0.82-1.69)$ & 0.376 & & \\
\hline Occupation & & & & & & \\
\hline Housewife & $67(66 \%)$ & $35(34 \%)$ & 1.00 & & & \\
\hline Factory worker & $35(46 \%)$ & $41(54 \%)$ & $2.24(1.22-4.12)$ & $<0.001$ & & \\
\hline Entertainment worker & $15(56 \%)$ & $12(44 \%)$ & $1.53(0.65-3.63)$ & & & \\
\hline Farmer & 29 (91\%) & $3(9 \%)$ & $0.20(0.06-0.70)$ & & & \\
\hline Employed & $57(58 \%)$ & $42(42 \%)$ & $1.41(0.80-2.50)$ & & & \\
\hline Self-employed/own business & $78(58 \%)$ & $56(42 \%)$ & $1.37(0.81-2.34)$ & & & \\
\hline Casual work/casual labour & $2(67 \%)$ & $1(33 \%)$ & $0.96(0.08-10.93)$ & & & \\
\hline Student & $5(29 \%)$ & $12(71 \%)$ & $4.59(1.50-14.09)$ & & & \\
\hline Unemployed & $4(44 \%)$ & $5(56 \%)$ & $2.39(0.60-9.48)$ & & & \\
\hline Other & $1(100 \%)$ & $0(0 \%)$ & $1.00(.-)$. & & & \\
\hline Contraception decision-making & & & & & & \\
\hline Mainly woman & $71(74 \%)$ & $25(26 \%)$ & 1.00 & & & \\
\hline Mainly husband/partner & $47(60 \%)$ & $31(40 \%)$ & $1.87(0.98-3.56)$ & 0.001 & & \\
\hline Joint decision & $154(53 \%)$ & $136(47 \%)$ & $2.51(1.50-4.18)$ & & & \\
\hline Phone sharing & & & & & & \\
\hline Shares & $142(59 \%)$ & $99(41 \%)$ & 1.00 & & & \\
\hline Never shares & $151(58 \%)$ & $108(42 \%)$ & $1.03(0.72-1.46)$ & 0.888 & & \\
\hline
\end{tabular}


Table 2 Associations with type of abortion (Continued)

\begin{tabular}{|c|c|c|c|c|c|c|}
\hline & Surgical & Medical & Crude OR & LRT $p$ value & Adjusted $\mathrm{OR}^{\mathrm{a}}$ & LRT $p$ value \\
\hline & $N=293$ & $N=207$ & & & & \\
\hline Disclosure of abortion to & & & & & & \\
\hline Yes & $122(60 \%)$ & $82(40 \%)$ & 1.00 & & & \\
\hline No & $20(54 \%)$ & $17(46 \%)$ & $1.26(0.63-2.56)$ & 0.515 & & \\
\hline PAFP intentions & & & & & & \\
\hline Yes & $135(72 \%)$ & $52(28 \%)$ & 1.00 & & & \\
\hline No & $22(52 \%)$ & $20(48 \%)$ & $2.36(1.19-4.68)$ & $<0.001$ & $2.08(1.02-4.23)$ & 0.001 \\
\hline Undecided & $136(50 \%)$ & $135(50 \%)$ & $2.58(1.73-3.84)$ & & $2.18(1.44-3.31)$ & \\
\hline Fertility plans & & & & & & \\
\hline No more/none & $105(76 \%)$ & $34(24 \%)$ & 1.00 & & & \\
\hline Have a/another child & $162(53 \%)$ & $146(47 \%)$ & $2.78(1.78-4.35)$ & $<0.001$ & $2.07(1.29-3.32)$ & 0.007 \\
\hline Undecided & $26(49 \%)$ & $27(51 \%)$ & $3.21(1.65-6.22)$ & & $2.08(1.03-4.18)$ & \\
\hline Phone credit & & & & & & \\
\hline Sometimes & $123(64 \%)$ & $70(36 \%)$ & 1.00 & & & \\
\hline Usually & $67(52 \%)$ & $61(48 \%)$ & $1.60(1.02-2.52)$ & 0.120 & & \\
\hline Always & $103(58 \%)$ & $76(42 \%)$ & $1.30(0.85-1.97)$ & & & \\
\hline
\end{tabular}

${ }^{a}$ Adjusted for factors included in the final model

contacted were considered lost to follow up and excluded from the analyses on contraceptive use, repeat pregnancy and repeat abortion.

Thirdly, we conducted an exploratory analysis to compare the timing of initiation of long-acting contraception between women having medical versus surgical abortion over the four-month post-abortion period. This analysis used data on weekly contraceptive use was obtained retrospectively at four-month follow-up.

Ethical approval for the MOTIF study was obtained from ethics committees at the London School of Hygiene and Tropical Medicine and Marie Stopes International and from the Cambodia Human Research ethics committee. All analyses were conducted using Stata, Version 15.

\section{Results}

Of the 500 women who participated in the trial $41 \%$ had a medical abortion. Over two thirds of the sample were aged over 25 , almost two thirds lived in rural areas, and the majority had access to motorized transport. Almost two thirds had secondary level education or above, and nearly all were married or living together. The full description of the sample is shown in Table 1. Table 2 shows the characteristics of women attending for medical versus surgical abortion. Univariable analyses suggested that younger age $(p=0.019)$, urban versus rural area of residence $(p<0.001)$, access to motorized transport $(p=0.058)$, higher level of education $(p=0.005)$, having no living children $(p=0.001)$, having mainly joint or mainly partner decision-making about contraception $(p=0.001)$, not intending or undecided about
PAFP $(p<0.001)$, plans to have another child or undecided about more children $(p<0.001)$, were associated with increased odds of medical versus surgical abortion. Occupation was associated with type of abortion $(p<0.001)$. During development of a final multivariable model, age was included first, then factors identified in univariable analyses were added and evaluated for association with abortion type after adjustment in turn, first looking at socio-economic factors and then fertility and contraceptive related factors. The final model included level of education, PAPF intentions, fertility plans and area of residence. Age and number of children were no longer associated with type of abortion after adjustment for contraceptive and fertility plans. After adjustment for factors included in the final model, medical versus surgical abortion was more likely amongst those with more education, those without plans for or undecided about PAFP, those undecided or desiring a child in future and those living in urban areas. Whether contraceptive decisions were mainly made by a husband/partner or made jointly was only asked to those who were currently married. Of this group of 464 women, joint or partner contraceptive decision making was not strongly associated with type of abortion after adjustment for other factors in the final model for all women $(p=0.085)$. After adjustment for the other variables in the final model, occupation was not associated with abortion type (LRT $p=0.289$ ).

Table 3 shows associations between abortion method and subsequent contraceptive use, repeat pregnancy and repeat abortion. Surgical abortion was associated with increased odds of using effective contraception at 4 months compared to medical abortion but this 
Table 3 Associations between abortion method and subsequent contraception use, repeat pregnancy and abortion

\begin{tabular}{|c|c|c|c|c|c|c|c|c|c|c|c|c|}
\hline \multicolumn{7}{|c|}{ Four month follow-up } & \multicolumn{6}{|c|}{12 month follow-up } \\
\hline & No & Yes & Crude OR & $\begin{array}{l}P \\
\text { value }\end{array}$ & Adjusted $\mathrm{OR}^{*}$ & $\begin{array}{l}P \\
\text { value }\end{array}$ & No & Yes & Crude OR & $\begin{array}{l}P \\
\text { value }\end{array}$ & Adjusted $O R^{*}$ & $\begin{array}{l}P \\
\text { value }\end{array}$ \\
\hline \multicolumn{13}{|c|}{ Effective contraception use } \\
\hline Medical & $93(52 \%)$ & $85(48 \%)$ & 1.00 & & & & 75 (54\%) & $63(46 \%)$ & 1.00 & & & \\
\hline Surgical & $\begin{array}{l}102 \\
(40 \%)\end{array}$ & $\begin{array}{l}151 \\
(60 \%)\end{array}$ & $\begin{array}{l}1.62 \\
(1.10-2.38)\end{array}$ & 0.015 & $1.44(0.95-2.18)$ & 0.084 & $101(53 \%)$ & $89(47 \%)$ & $\begin{array}{l}1.05 \\
(0.68-1.63)\end{array}$ & 0.831 & $0.87(0.55-1.39)$ & 0.563 \\
\hline \multicolumn{13}{|c|}{ Long-acting contraception use } \\
\hline Medical & $\begin{array}{l}146 \\
(82 \%)\end{array}$ & $32(18 \%)$ & 1.00 & & & & $113(82 \%)$ & $25(18 \%)$ & 1.00 & & & \\
\hline Surgical & $\begin{array}{l}205 \\
(81 \%)\end{array}$ & $48(19 \%)$ & $\begin{array}{l}1.07 \\
(0.65-1.75)\end{array}$ & 0.794 & $0.87(0.51-1.49)$ & 0.611 & $154(81 \%)$ & $36(19 \%)$ & $\begin{array}{l}1.06 \\
(0.60-1.86)\end{array}$ & 0.848 & $0.84(0.46-1.54)$ & 0.573 \\
\hline \multicolumn{13}{|c|}{ Repeat pregnancy } \\
\hline Medical & $\begin{array}{l}171 \\
(97 \%)\end{array}$ & $6(3 \%)$ & 1.00 & & & & $116(84 \%)$ & $22(16 \%)$ & 1.00 & & & \\
\hline Surgical & $\begin{array}{l}248 \\
(98 \%)\end{array}$ & $5(2 \%)$ & $\begin{array}{l}0.57 \\
(0.17-1.91)\end{array}$ & 0.367 & $0.82(0.23-2.86)$ & 0.750 & $162(85 \%)$ & $28(15 \%)$ & $\begin{array}{l}0.91 \\
(0.50-1.67)\end{array}$ & 0.764 & $0.97(0.51-1.84)$ & 0.924 \\
\hline \multicolumn{13}{|c|}{ Repeat abortion } \\
\hline Medical & $\begin{array}{l}176 \\
(99 \%)\end{array}$ & $1(1 \%)$ & 1.00 & & & & $127(92 \%)$ & $11(8 \%)$ & 1.00 & & & \\
\hline Surgical & $\begin{array}{l}251 \\
(99 \%)\end{array}$ & $2(1 \%)$ & $\begin{array}{l}1.40 \\
(0.13-15.59)\end{array}$ & 0.783 & $2.04(0.17-24.22)$ & 0.572 & $182(96 \%)$ & $8(4 \%)$ & $\begin{array}{l}0.51 \\
(0.20-1.30)\end{array}$ & 0.157 & $0.50(0.19-1.35)$ & 0.172 \\
\hline
\end{tabular}

*Adjusted for age, residence, socio-economic status and whether randomised

association did not remain after adjusting for confounding variables (aOR 1.44; 95\%CI 0.95-2.18). There were no statistically significant differences in contraceptive use, repeat pregnancy or repeat abortion comparing women having medical versus surgical abortion, but it is acknowledged that power was low for these analyses.

Figure 1 shows the timing of initiation of long-acting contraception over the four-month post-abortion period. Women having surgical abortion appear more likely to initiate long-acting contraception sooner compared to women having medical abortion; 50 women reported initiating long-acting contraceptive after surgical abortion, of whom $34(68 \%)$ started during the first week. 29 women reported initiating long-acting contraception use after medical abortion, of whom nine (31\%) started during the first week $(p=0.001)$. Of the 34 women who had a surgical abortion and started using long-acting contraception during the first week, 14 (41\%) started using an IUD and 20 started using the implant. Of the nine women who had a medical abortion and started using long-acting contraception during the first week, two (22\%) started using an IUD and seven started using the implant.

\section{Discussion}

\section{Summary of main results}

In this observational study using randomized controlled trial data, women having a medical abortion were more likely than those having a surgical abortion to be of urban residence, more educated, not planning to use or undecided about PAFP, and be planning to have another child or undecided. There was no association between type of abortion (medical vs. surgical) and use of effective contraception at 4 months, repeat pregnancy, or repeat abortion. Initiation of long-acting contraception within the first week was less likely with medical than surgical abortion.

\section{Strengths and weaknesses}

To our knowledge, this is the first quantitative study reporting differences in characteristics of medical versus surgical abortion clients attending non-governmental health facilities in Cambodia, and complements trends in medical abortion use observed in the Demographic and Health Survey and previous qualitative studies in Cambodia and neighbouring Vietnam. $[2,5,20]$ The study benefits from a sample size of 500 and a follow-up period of 12 months with good follow-up rates.

A key limitation of the study is the potential for residual and unmeasured confounding. It would have been particularly useful to have data on pregnancy duration. The 2014 Cambodian Demographic and Health Survey reported that use of surgical abortion increased with age and pregnancy duration [3, 7]. We also do not have information on provider behaviors - it is possible that providers may show bias in the type of abortion that they offer or recommend. Furthermore, we cannot draw 


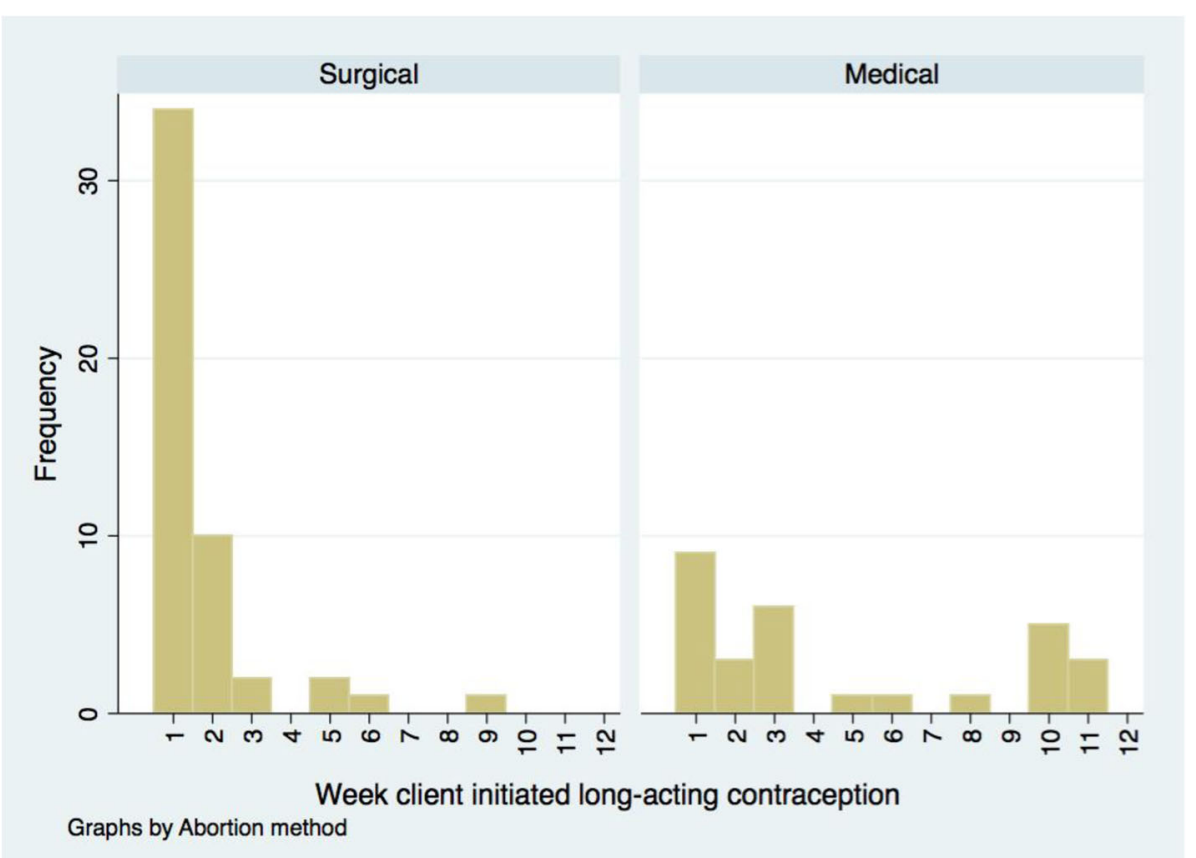

Fig. 1 Initiation of long-acting contraception (IUD or implant) over 4-month post-abortion period *data obtained retrospectively at 4 months asking participants about weekly contraceptive use. 50 women reported initiating long-acting contraceptive after surgical abortion, of whom 34 (68\%) started during the first week. 29 women reported initiating long-acting contraception use after medical abortion, of whom 9 (31\%) started during the first week

conclusions on the direction of associations and causality.

A further limitation concerns the generalizability of the findings. These results reflect the NGO clinic population and may be different in public and private health facilities, and among women obtaining medical abortion from pharmacies. However, the proportions obtaining medical abortion are similar to 2013 data from MSI Cambodia. [14] In addition, the sample was restricted to women who had access to a mobile phone, although in Cambodia over 90\% of women are estimated to own a mobile phone [21]. Future research should examine the needs of women using MA outside of the formal heath sector (e.g. from drug sellers) in terms of follow-up and PAFP. Further qualitative research could also consider how fertility intentions and provider biases might influence both the type of abortion that a woman has and subsequent use of PAFP.

Finally, the study was underpowered to examine differences in contraceptive and reproductive outcomes between women having medical and surgical abortions although we found no evidence of a difference in PAFP uptake, repeat pregnancy or repeat abortion in this sample, a larger study may do.

\section{Discussion of the findings in context of existing research} Previous research has shown that a wide range of socio demographic variables as well as knowledge of abortion methods influence the uptake of medical abortion rather than surgical abortion. Our finding that rural women were less likely to choose MA are consistent with findings from Ethiopia, India, and Nepal but not Vietnam [22-25]. Our finding that educated women were more likely to use MA are in keeping with study findings in the USA and Nepal, but studies in Ethiopia and Vietnam did not find such an association [24-26]. To our knowledge no previous studies have reported the association between choice of MA and uncertainty about future contraceptive and fertility plans.

Access to a clinic is a barrier to obtaining abortion services and poor geographic access has been shown to be a barrier to women seeking other maternal health services in rural areas of Cambodia [27, 28]. Our finding that women living in rural areas are more likely to opt for surgical abortion rather than medical abortion could be due to the distance to clinic and the desire for greater certainty about the abortion being complete at the time of the clinic visit. It may also be that greater difficulties in access result in rural women attending later for medical abortion.

In this setting, women were offered a follow-up visit to check for post-abortion complications or a continuing pregnancy after both medical and surgical abortion as per national guidelines, [13] although routine data show that many women do not attend. [29] MSI Cambodia 
reports a 97.3\% success rate for medical abortion. Self-assessment of the outcome of medical abortion is accurate, feasible and safe in low-resource settings. [30, 31] Enabling women to self-manage follow-up, as recommended in the WHO guidelines, [32] is likely to reduce barriers to medical abortion in urban and rural low-resource settings. [33].

Women with more education might be more confident in using medical abortion as it might be easier for them to read any written instructions provided and contact the clinic in case of a problem.

The associations between abortion type and contraception and fertility intentions could be explained by concerns about future fertility. Women who were undecided or planning on having more children (i.e. birth spacers rather than limiters) may have a preference for medical abortion if surgical abortion or contraception use is perceived as more harmful, potentially affecting the uterus and impacting fertility. [4]

Women undergoing medical abortion were no less likely than those undergoing surgical abortion to obtain post-abortion family planning (PAFP), including long-acting reversible contraception (LARC). All participants received standard care at the clinic prior to randomization, which included contraceptive counselling and the choice of a range of methods (e.g. pill, injectable and implant), all of which could be obtained on the day. It is unsurprising that women obtaining MA have lower uptake of IUD than women having surgical abortion as women could have an IUD inserted during surgical procedure, while women undergoing medical abortion have to attend and appointment for fitting 2 weeks later. It is perhaps surprising that women undergoing surgical abortion were not more likely to use LARC given the convenience of IUD insertion during the procedure, versus a repeat visit following medical abortion. However, LARC use is relatively low in Cambodia; roughly $2 \%$ of married women use the implant and $4 \%$ use the IUD compared to $9 \%$ who use the injectable, and $18 \%$ who use the contraceptive pill [3]. In contexts with a different baseline method mix, there might be greater differences in uptake of LARC between those undergoing medical and surgical abortion.

The findings did show that among women who started LARC methods post-abortion, women who obtained a medical abortion had a more delayed initiation. This may reflect the delay required between the procedure and the insertion of an IUD following medical abortion. This is supported by the finding that, among those who started LARC in the first week, the proportion starting to use an IUD (rather than implant) was higher among women who had a surgical abortion. Additionally, women having a medical abortion may have wished to have confirmation that the abortion was complete before starting to use the implant.

\section{Conclusion}

Our results suggest women having a medical abortion in Cambodia are more likely to be educated and live in urban areas and had delayed uptake of contraception compared to women having a surgical abortion, although there was not strong evidence for a difference in contraceptive use at four or 12 month follow up. Our findings suggest there is a continuing need to improve access to abortion services, particularly in rural areas, as well as to take into account the factors influencing women's choice of abortion method during the consultation. They also raise questions about whether routine follow-up is required after abortion. Enabling self-management of medical abortion follow up may increase women's choice around the procedure by enabling women who would prefer medical abortion but cannot because of loss of earnings or distance barriers to do so Further research is recommended to increase access to abortion and post-abortion contraception.

\section{Acknowledgements \\ We thank all clients and clinic staff who participated in the study; Channe Suy at the InSTEDD iLab South East Asia; and Uk Vannak, Ly Sokhey, Sieklot Chinn, Melissa Cockcroft, Nicky Jurgens, Michelle Phillips, Sras Thorng, Stefanie Wallach and Amy Williamson at Marie Stopes International. \\ Funding \\ The Marie Stopes International Innovation Fund funded the study for 15 months from October 2012 and had some influence on the study design but not on data collection or analysis. The UK Medical Research Council (MRC) funded the data analysis but had no influence on the analysis or reporting. Chris Smith was supported by an MRC Population Scientist Fellowship, grant reference MR/L012251/1 (April 2014 to September 2018).}

\section{Availability of data and materials}

Data are from the MOTIF study whose authors may be contacted at the London School of Hygiene and Tropical Medicine, Keppel Street, London, WC1E7HT. Email: christopher.smith@lshtm.ac.uk.

\section{Authors' contributions}

CS conceived of the current study, CS, RS and TE analysed and interpreted the data. CS and RS wrote successive drafts. CF commented on some of the drafts. All authors reviewed, contributed to and approved the final version for publication.

\section{Ethics approval and consent to participate}

Participants provided consent by written signature or thumbprint. Ethical approval was obtained from ethics committees at the London School of Hygiene \& Tropical Medicine and Marie Stopes International and the Cambodia Human Research ethics committee. The trial was registered through ClinicalTrials.gov with the identifier NCT01823861.

Consent for publication

Not applicable.

\section{Competing interests}

The authors declare that they have no competing interests.

\section{Publisher's Note}

Springer Nature remains neutral with regard to jurisdictional claims in published maps and institutional affiliations.

\section{Author details}

${ }^{1}$ Graduate School of Tropical Medicine and Global Health, Nagasaki

University, Nagasaki, Japan. ${ }^{2}$ Department of Population Health, London 
School of Hygiene \& Tropical Medicine, London, UK. ${ }^{3}$ MRC Tropical Epidemiology Group, London School of Hygiene \& Tropical Medicine, London, UK.

Received: 25 July 2018 Accepted: 24 April 2019

Published online: 16 May 2019

\section{References}

1. Sedgh G, Singh S, Hussain R. Intended and unintended pregnancies worldwide in 2012 and recent trends. Stud Fam Plan. 2014;45:301-14. https://doi.org/10.1111/j.1728-4465.2014.00393.x.

2. Petitet $\mathrm{PH}$, Ith L, Cockroft M, Delvaux T. Towards safe abortion access: an exploratory study of medical abortion in Cambodia. Reprod Health Matters. 2015;22:47-55. https://doi.org/10.1016/S0968-8080(14)43826-6.

3. National Institute of Statistics: Directorate General for Health and ICF International. Cambodia Demographic and Health Survey 2014 [Internet]. 2015. Available from: https://dhsprogram.com/pubs/pdf/FR312/FR312.pdf.

4. Hemmings J, Rolfe B. Abortion in cambodia [Internet]. Options; 2008. Available from: www.options.co.uk.

5. Petitet P, Desclaux A. Reproductive health and HIV in Cambodia: from anthropology to public health. Aix Marseille III (France): Groupe de Recherche cultures, Santé, Sociétés, Paul Cézanne Université; 2011.

6. National Institute of Public Health: National Institute of Statistics and ORC Macro. Cambodia Demographic and Health Survey 2005. 2006;487. Available from: https:/dhsprogram.com/pubs/pdf/FR185/FR185\%5BApril-27-2011\%5D.pdf.

7. National Institute of Statistics: Directorate General for Health and ORC Macro. Cambodia Demographic and Health Survey 2010 [Internet]. 2010. Available from: http://www.measuredhs.com/pubs/pdf/FR249/FR249.pdf.

8. USAID. High Impact Practices in Family Planning (HIP). Postabortion family planning: strengthening the family planning component of postabortion care. Washington DC: USAID; 2012

9. Stoddard A, Eisenberg DL. Controversies in family planning : timing of ovulation after abortion and the conundrum of postabortion intrauterine device insertion. Contraception. 2011;84:119-21. https://doi.org/10.1016/j. contraception.2010.12.010.

10. USAID. Post-Abortion family planning: a key component of Post abortion care. 2013.

11. World Health Organization. Medical eligibility criteria for contraceptive use; 2015. https://doi.org/10.2165/00128413-200112870-00010.

12. Cameron S. Postabortal and postpartum contraception. Best Pract Res Clin Obstet Gynaecol. 2014;28:871-80. https://doi.org/10.1016/j.bpobgyn. 2014.05.007.

13. Cambodia Ministry of Health. National Protocol Cambodia: comprehensive abortion care 2010

14. Smith C, Ngo T, Gold J, Edwards P, Vannak U, Sokhey L, et al. Effect of a mobile phone-based intervention on post-abortion contraception: a randomized controlled trial in Cambodia. Bull World Health Organ. 2015;93: 842-50. https://doi.org/10.2471/BLT.15.160267.

15. Smith C, Ly S, Uk V, Edwards P, Warnock R, Free C. Process evaluation of a mobile phone based intervention to support post-abortion contraception in Cambodia. Contracept Reprod Med. 2017;16. https://doi.org/10.1186/ s12978-017-0329-y.

16. Smith C, Vannak U, Sokhey L, Ngo TD, Gold J, Khut K, et al. MObile Technology for Improved Family Planning services (MOTIF): study protocol for a randomised controlled trial. Trials. 2013;14(427):1-9.

17. Smith C, Jarvis C, Free C. Assessing loss to follow-up in the MObile Technology for Improved Family Planning (MOTIF) randomised controlled trial. Trials. 2017;18:577. https://doi.org/10.1186/s13063-017-2316-6.

18. World Health Organization. Family planning: a global handbook for providers. 2011

19. Maries Stopes International. Young people [Internet]. Available from: https:// mariestopes.org/who-we-serve/young-people/. [cited 2019 Jan 15].

20. Ngo TD, Free C, Le HT, Edwards P, Pham KHT, Nguyen YBT, et al. Women's perspectives on termination service delivery in Vietnam: a cross-sectional survey in three provinces. Int J Women's Health. 2014;6:927-33. https://doi. org/10.2147/IJWH.S72343.

21. Kimchhoy P, Sola J. Mobile Phones in Cambodia 2014 [Internet]. 2014. Available from: https://asiafoundation.org/resources/pdfs/MobilephonesinCB.pdf.

22. Tamang A, Tuladhar S, Tamang J, Ganatra B, Dulal B. Factors associated with choice of medical or surgical abortion among women in Nepal. Int J Gynecol Obstet. 2012;118:52-6. https://doi.org/10.1016/j.ijgo.2012.05.011.
23. Das $\mathrm{V}$. Evaluation of newer methods of early pregnancy termination. J Obs Gynecol India. 2005;55:454-6.

24. Woldetsadik MA, Sendekie TY, White MT, Zegeye DT. Client preferences and acceptability for medical abortion and MVA as early pregnancy termination method in Northwest Ethiopia. Reprod Health. 2011;9:4-8.

25. Ngo TD, Free C, Le HT, Edwards P, Pham KHT, Nguyen YBT, et al. Service users' attributes associated with the uptake of medical versus surgical abortion at public health facilities in Vietnam. Int J Gynecol Obstet. 2014; 125:247-52.

26. Clark S. Is medical abortion acceptable to all American women: the impact of sociodemographic characteristics on the acceptability of mifepristonemisoprostol abortion. J Am Med Womens Assoc. 2012;5:177-82.

27. Matsuoka S, Aiga H, Chan L, Rathavy T, Okitsu A. Perceived barriers to utilization of maternal health services in rural Cambodia. Health Policy. 2010; 95:255-63. https://doi.org/10.1016/j.healthpol.2009.12.011.

28. Yanagisawa $S$, Oum S, Wakai S. Determinants of skilled birth attendance in rural Cambodia. Trop Med Int Heal. 2006;11:238-51. https://doi.org/10.1111/ j.1365-3156.2005.01547.x

29. Smith C, Vannak U, Sokhey L, Ngo TD, Gold J, Free C. Mobile Technology for Improved Family Planning (MOTIF): the development of a mobile phonebased (mHealth) intervention to support post-abortion family planning (PAFP) in Cambodia. Reprod Health. 2016;13:1-8. https://doi.org/10.1186/ S12978-015-0112-X.

30. Blum J, Sheldon WR, Ngoc NTN, Winikoff B, Nga NTB, Martin R, et al. Randomized trial assessing home use of two pregnancy tests for determining early medical abortion outcomes at 3, 7 and 14 days after mifepristone. Contraception. 2016;94(2):115-21. https://doi.org/10.1016/j. contraception.2016.04.001

31. lyengar K, Paul M, lyengar SD, Klingberg-Allvin M, Essén B, Bring J, et al. Selfassessment of the outcome of early medical abortion versus clinic follow-up in India: a randomised, controlled, non-inferiority trial. Lancet Glob Heal. 2015;3:e537-45. https://doi.org/10.1016/S2214-109X(15)00150-3.

32. WHO. Clinical practice handbook for safe abortion. 2014.

33. Sheldon WR. Home use of urine pregnancy tests for medical abortion follow-up. Lancet Glob Heal. 2015;3:e504-5. https://doi.org/10.1016/S2214109X(15)00153-9.

\section{Ready to submit your research? Choose BMC and benefit from:}

- fast, convenient online submission

- thorough peer review by experienced researchers in your field

- rapid publication on acceptance

- support for research data, including large and complex data types

- gold Open Access which fosters wider collaboration and increased citations

- maximum visibility for your research: over 100M website views per year

At BMC, research is always in progress.

Learn more biomedcentral.com/submissions 